\title{
Sub-Gigahertz Inter-Technology Interference. How Harmful is it for LoRa?
}

\author{
Jetmir Haxhibeqiri*, Adnan Shahid*, Martijn Saelens*, Jan Bauwens*, \\ Bart Jooris*, Eli De Poorter*, and Jeroen Hoebeke* \\ * IDLab, Ghent University - imec, Ghent, Belgium \\ Email: jetmir.haxhibeqiri@ugent.be
}

\begin{abstract}
Sub-one $\mathbf{G H z}$ technologies are getting utilized for different applications in home automation and smart cities, due to their long coverage range and support for large number of end devices. Due to different technologies used by different applications and device vendors, the impact of each technology on each other needs to be assessed in environments where multiple technology are used and most of the time they are coexisting with each other. In this paper, we show an inter-technology interference measurement setup for LoRa, Sigfox, Z-Wave and IO Home Control. In the setup, the packet transmissions are controllable in time and frequency, that makes it possible to test and evaluate various interference scenarios. From the designed and implemented setup, we evaluate the impact of the sub-GHz technologies (Sigfox, Z-wave, and IO Home Control) on LoRa. The results show that there is a significant loss (up to $20 \%$ ) and a relatively lower loss (up to $12-15 \%$ ) under Sigfox and Z-Wave or IO Home Control, respectively, when the interferer starts during the preamble and header time. Losses are practically zero if the interferer starts during the payload time.
\end{abstract}

Index Terms-LoRa, SigFox, IO Home Control, Z-Wave, interference measurements, interference.

\section{INTRODUCTION}

Sub-one GHz technologies are getting utilized for different applications in home automation and smart city domains. Their wide coverage zone makes them a potential candidate for applications (like smart cities), where end devices are far apart from the network. On the other hand, in home automation domain, sub-one $\mathrm{GHz}$ technologies are the preferred choice due to low penetration losses of the building materials [1]. With densification of applications and end devices from different vendors that use different technologies, problems caused by inter-technology interference increases.

From the vendor perspective, it is crucial to know the impact of one technology over the others before selecting the right technology for their products. On the other hand, if a technology is already deployed in an environment, it is beneficial for the developers to know what is the impact of the newly deployed technology on the already deployed one.

Different characteristics of each technology can be taken into account beforehand. LoRa technology is a wide spread spectrum technology, that has low receiver sensitivity (up to $-140 \mathrm{dBm}$ ) which makes it possible to receive packets below noise floor. On the other hand, SigFox is a narrow band technology that concentrates the transmit power in a single narrow band, that decreases the possibility to get interfered from other coexisting technologies. Looking from the time domain perspective, LoRa has a shorter on-air packet time compared to SigFoX, but both of them have quite a long onair packet time compared to other technologies due to low bit rates. This increases the chance for a packet to get interfered as the transmission time is long.

In order for different technologies to co-exists interference on one-another has to be assessed. In this paper, we assess the SigFox, Z-Wave and IO Home Control interference on LoRa. By means of a controllable setup, we do a set of measurements for different power differences between LoRa and the interferer technology as well as different time shifts between transmission of LoRa packet and the interferer packet. LoRa channels that are used are based on the operational channels of the interfering technology, being as near as possible with the LoRa channels. While for SigFox a TDnext RF module [2] was used, for the other two interfering technology emulated versions on Zolertia Remotes were used.

The rest of the paper is organized as follows: section II gives the related work in the interference measurements and sub$\mathrm{GHz}$ spectrum occupancy while section III gives an insight to characteristics of the considered technologies (LoRa, SigFox, Z-Wave and IO Home Control). Section IV describes the measurement setup, methodology of measurements as well as the way how the technologies (Z-Wave and IO Home Control) were emulated, while section $\mathrm{V}$ shows measurement results. Finally, section VI concludes the paper.

\section{RELATED WORK}

There have been a number of studies that evaluate the impact of interference on LoRa performance, mostly being the result of self-interference and imperfection of spreading factor (SF) orthogonality.

In [3] differences between ultra-narrow band (UNB) and wide spread spectrum technologies in terms of range and resistance against interference are studied. By means of a network simulator authors in [3] show that the UNB networks cope well with wide spread spectrum network interference, while the reverse is valid for the vice-verse case. It means that in case of coexistence of LoRa and Sigfox, LoRa network will have higher losses due to SigFox interference, being up to $50 \%$ for LoRa end nodes at the cell edge. In [4] a performance comparison between different physical technologies (UNB, chirp spread spectrum (CSS) and Weightless) used in Internet of Things (IoT) is done. For LoRa self-interference, in case 
of interference by the same spreading factor signal, the packet that is received with $6 \mathrm{~dB}$ higher signal strength will be received correctly. In addition, authors show the co-channel rejection for all combinations of spreading factors in case of LoRa. Considering the non-LoRa interference, LoRa can cope with a single-tone pulse interference if it is less than $5 \mathrm{~dB}$ (19.5 dB) above the desired signal for SF7 (SF12) [4].

In [5] impact of spreading factor orthogonality imperfection in interference is shown. The higher spreading factors (10 to 12) are more prone to get interfered by other spreading factors. As higher spreading factors will be used for far away end nodes to increase receiver sensitivity, they will be impacted by the near-far problem. However, by deploying multiple gateways the impact can be alleviated as different gateways experience different Signal-to-Interference Ratio (SIR) values [5].

In [6] the impact of interference between LoRa and IEEE 802.15.4g on each another is shown. Based on empirical measurement, for different power levels and different spreading factors, LoRa is shown to have high packet reception ratio (PRR) under IEEE 802.15.4g interference. Always for spreading factors higher than SF9 PRR is one, no matter the configuration of IEEE $802.15 .4 \mathrm{~g}$ channel usage or power level. In case of SF7 the PRR drops around 0.4 if IEEE 802.15.4 operates at channel 26.

In [7] authors study the effect of spreading factors of the transmitter and the interferer on the packet success ratio. By means of empirical measurements it is shown that higher SFs are more prone to get interfered than lower SFs due to higher time on air. On the other hand, when two signals are transmitted using the same SF, at least one of them can be captured with high probability $(>80 \%)$ if it is $6 \mathrm{~dB}$ stronger. Moreover, it is shown that the LoRa modulated signals are interfered by Gaussian frequency shift keying (GFSK) modulated signals. In [8] LoRa communication performance in an industrial environment is shown.

\section{Introduction to CONSIDERED TeChNOLOGIES}

In this section we will describe different characteristics of the considered technologies. We will cover characteristics such as: modulation techniques they use, channels, channel bandwidths, data rates etc.

\section{A. LoRa}

LoRa is wide spread spectrum based technology that represents each symbol of information by multiple chips. The number of chips per symbol is determined based on the spreading factor and it is $2^{S F}$, where $\mathrm{SF}$ is the spreading factor used. The spreading factor can be from 7 to 12 . By increasing the spreading factor the nominal data rate is decreased, as more chips are sent for single information symbol. However, this will increase the receiver sensitivity as well as the robustness against interference. E.g for SF12 the receiver sensitivity will be $-137 \mathrm{dBm}$ compared to $-124 \mathrm{dBm}$ for SF7 [9]. Table I shows the nominal data rates and receiver sensitivities per spreading factor. The froward error correction (FEC) codes are used to
TABLE I

NOMINAL DATA RATE AND RECEIVER SENSITIVITY FOR DIFFERENT SF.

\begin{tabular}{|c||c|c|}
\hline SF & Bit rate [kbps] & Rx sensitivity [dBm] \\
\hline \hline 12 & 0.25 & -137 \\
\hline 11 & 0.44 & -135 \\
\hline 10 & 0.98 & -133 \\
\hline 9 & 1.7 & -130 \\
\hline 8 & 3.1 & -129 \\
\hline 7 & 5.4 & -124 \\
\hline
\end{tabular}

detect and correct bit errors. Code rates from $4 / 5$ to $1 / 2$ are used. To be even more protective against burst noise, diagonal interleaving is used to spread the information symbols over time [10].

LoRa physical frame is composed by the preamble, physical header and the payload. The preamble is used to synchronize the receiver with the transmitter before actually starting to decode the data. The preamble starts with a programmable sequence of upchirps, that can be 6 to 65535 symbols long. The programmable part of the preamble is followed by two upchirps that encode the sync word and ends with 2.25 downchirps [9]. Physical header can be optional. If it is present in the packet it contains the information about the payload length and code rate of the payload. It is always protected by the FEC codes with code rate of $1 / 2$ and has its own cyclic redundancy check (CRC) for header integrity checkup. The payload can be encoded with different code rate than header and can have an optional payload CRC.

In Europe, LoRa operates in the $863-870 \mathrm{MHz}$ frequency band. It can operate in two sub-bands, one at $868 \mathrm{MHz}$ that offers three $125 \mathrm{KHz}$ LoRa channels and one at $867 \mathrm{MHz}$ that offers five $125 \mathrm{kHz}$ LoRa channels. Three channels at $868 \mathrm{MHz}$ have center frequencies at $868.1 \mathrm{MHz}, 868.3 \mathrm{MHz}$ and $868.5 \mathrm{MHz}$.

\section{B. Sigfox}

SigFox is a proprietary technology and operates on different spectrum bands in Europe and in the US: $868 \mathrm{MHz}$ and $902 \mathrm{MHz}$, respectively. It utilizes ultra-narrow bandwidth modulation scheme, which is also termed as Differential Phase Shift Keying (DPSK) in uplink and Gaussian Frequency Shift Keying (GFSK) in downlink. Due to the narrow band in the uplink, quite a large number of devices can communicate over a distance of 10 to $50 \mathrm{~km}$. However, a downside is that it can only achieve up to 100bps data rate [11]. In addition to low data rate, there is also a regional regulation of $1 \%$ duty cycle [12]. Thus, the duty cycle limitation imposes a daily limit on a Sigfox device with a maximum 140 messages in uplink and 4 messages in downlink. The maximum payload in uplink and downlink is 12 bytes and 8 bytes, respectively.

\section{Z-Wave}

Z-Wave is a ITU-T G.9959 standard [13] based low-power wireless technology that is used in home automation for connecting different sensors. ITU-T G.9959 specifies a list of 
radio frequency (RF) profiles that each node has to comply to, depending on the region of operation. The RF profile is a combination of center frequencies and data rates. Three different data rates are supported 9.6 Kbps (R1), $40 \mathrm{Kbps}$ (R2) and $100 \mathrm{Kbps}$ (R3). Z-Wave operates at ISM bands, respectively at $868.40 \mathrm{MHz}$ and $869.85 \mathrm{MHz}$ band in Europe and $908.42 \mathrm{MHz}$ and $916 \mathrm{MHz}$ in the US.

Physical layer employs frequency shift keying (FSK) for the RF profiles R1 and R2 and Gaussian frequency shift keying (GFSK) for R3, respectively, as modulation techniques. Different coding schemes are used, Manchester encoding for R1 and non-return to zero (NRZ) encoding for R2 and R3. Receiver sensitivity typically is below $-104 \mathrm{dBm}$, while coverage can go up to $100 \mathrm{~m}$ in the line of sight communication or up to $30 \mathrm{~m}$ in an indoor home environment.

The physical layer frame format of a Z-Wave transmission is composed of a variable length preamble sequence, 1 byte start of frame delimiter, variable length of physical layer payload and in case of R1 profile 1 byte of end-of-frame delimiter. The preamble sequence is a variable byte sequence of the binary pattern "01010101" [13] that allows receiver to obtain symbol synchronization. The maximal physical layer payload is 64 bytes.

\section{IO Home Control}

Another technology that operates in sub-one $\mathrm{GHz}$ band is IO Home Control. It operates at $868 \mathrm{MHz}$ ISM band using one of three channels centered at $868.25,868.95,869.85 \mathrm{MHz}$. It uses FSK or GFSK as modulation techniques with an data rate of $38.4 \mathrm{Kbps}$ [14] .

\section{MEASUREMENT SETUP AND METHOdOLOGY}

The interference has different impacts based on the power strength of the interferer, the time how long the interference happens and the time when the interference started referring to the main transmission. We created a measurement setup where all of these three parameters can be controlled that can be used for any interference testing scenarios. The setup consists of a microcontroller that manages the time of transmissions of the devices (technologies) under test. Both devices are connected to two different controllable attenuators. After attenuating the signals coming from the devices (emulating the power level difference accordingly), both signals are combined and are sent to the receiver of the technology that is being tested, in our case LoRa receiver. All devices (transmitters and the receiver) are located inside the RF shielded boxes, in order to have no impact from any outsider transmitter and to have full control on introduced interference. In Figure 1 measurement setup schematic is shown. The Qosmotec boxes were used as $\mathrm{RF}$ shielded boxes. The attenuation box was of type PAH6000/80-2 [15] with eight input/output ports with maximal insertion losses of $14 \mathrm{~dB}$. The splitter/combiner box was from MTS Systemtechnik with eight paths with maximal insertion losses of $6 \mathrm{~dB}$ [16].

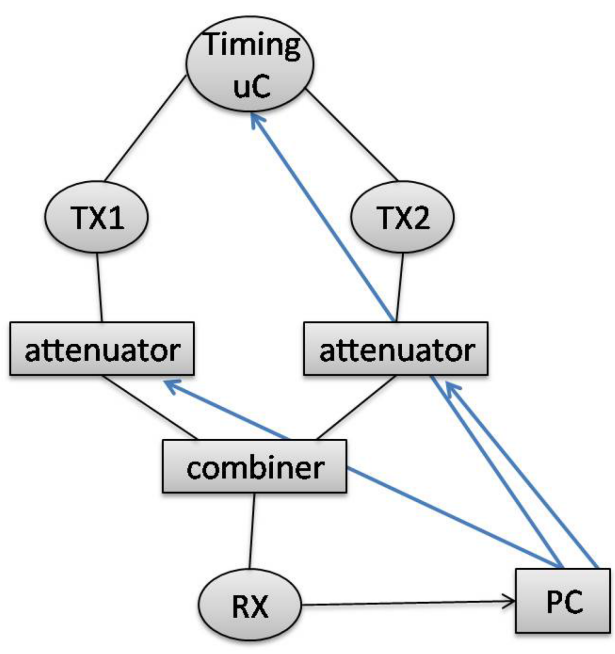

Fig. 1. Controllable measurement setup schematic.

\section{A. Methodology}

In typical home deployment setup end devices are not far apart from the gateway that is installed inside the house. So, compared to interfering technology that is already deployed in the neighborhood, the signal power of the main transmission always will be higher at the receiver side. In the reverse case, when both technologies are co-located and the interferer transmitter is nearer to the receiver than the main transmitter, the main transmitter can end up in lower received power than the interferer in the received side. To emulate these two scenarios we will test for two different power levels: when the interferer has $10 \mathrm{~dB}$ lower signal strength than the main transmission in the receiver side and when interferer is $10 \mathrm{~dB}$ higher. In our case the main transmission will be LoRa transmission while the other technologies will be the interferers. We set the received signal strength for LoRa at $-65 \mathrm{dBm}$, while the interferer signal strength at the receiver will be $+10 \mathrm{~dB}$ and $-10 \mathrm{~dB}$, respectively, compared to LoRa signal. This is achieved by adjusting the attenuation levels of the tunable attenuators. In Table II LoRa settings used for the measurements are shown. Channel centered at $868.1 \mathrm{MHz}$ was used for the measurements under SigFox interference while channel centered at $868.3 \mathrm{MHz}$ was used for measurements under Z-Wave and IO Home Control interference, in order to test the worst case scenario.

As it was shown in [17], interference has different impact on different LoRa packet parts. If the interference happens during the preamble it prevents the receiver to detect the packet. If it happens during the header time it makes the packet to be decoded wrongly due to wrong payload length, while if it happens during the payload the impact might be lower due to FEC code usage and diagonal interleaving. Based on this, different time shifts for the interferer after the start of the main transmission were used. The interference was started during the preamble time (time shifts of $8 \mathrm{~ms}$ for SF7 and $150 \mathrm{~ms}$ for SF12), during the header time (time shifts of $17 \mathrm{~ms}$ for SF7 


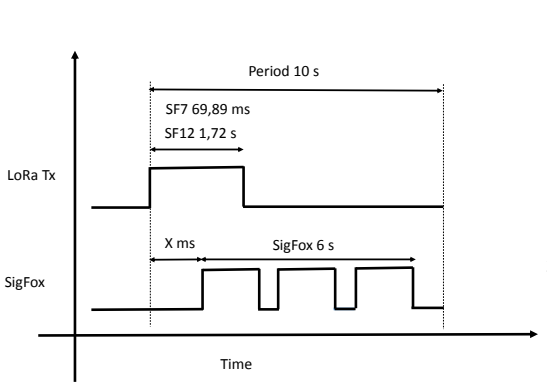

(a) SigFox. Measurement time 16 minutes.

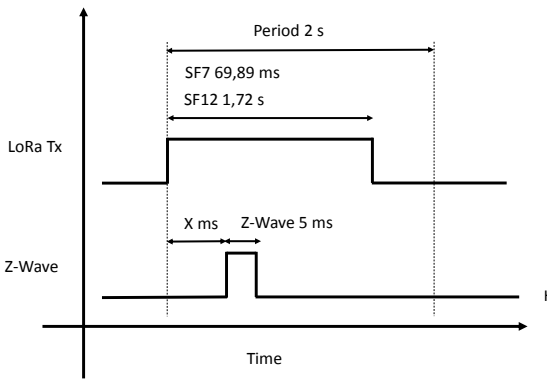

(b) Z-Wave. Measurement time 4 minutes.

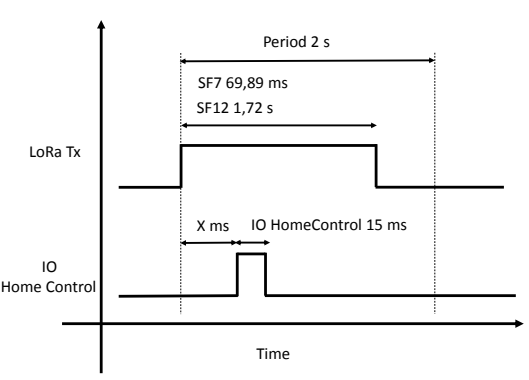

(c) IO Home Control. Measurement time 4 minutes.

Fig. 2. Different time shifts for different measurements. In case of SF7 $X=\{8,17,30,55\} \mathrm{ms}$ and in case of SF12 $X=\{150,500,1000,1500\} \mathrm{ms}$.

TABLE II

LORA SETTING FOR MEASUREMENTS

\begin{tabular}{|c||c|}
\hline Parameter & LoRa settings \\
\hline \hline Channels & $868.1,868.3 \mathrm{MHz}$ \\
\hline Bandwidth & $125 \mathrm{KHz}$ \\
\hline Data Rate & $5.4 \mathrm{Kbps}(\mathrm{SF}=7)$ and $183.11 \mathrm{bps}$ (SF12) \\
\hline Tx Power & $14 \mathrm{dBm}$ \\
\hline Payload length & 17 bytes \\
\hline Time on air & $69.89 \mathrm{~ms}(\mathrm{SF} 7) 1.72 \mathrm{~s} \mathrm{(SF12)}$ \\
\hline Physical header & Explicit \\
\hline Preamble time & $12.54 \mathrm{~ms}(\mathrm{SF} 7) 401.4 \mathrm{~ms}(\mathrm{SF} 12)$ \\
\hline Header time & $21.54 \mathrm{~ms}(\mathrm{SF} 7) 605.4 \mathrm{~ms}(\mathrm{SF} 12)$ \\
\hline Symbol time & $1.02 \mathrm{~ms}(\mathrm{SF} 7) 32.77 \mathrm{~ms}$ (SF12) \\
\hline
\end{tabular}

and $500 \mathrm{~ms}$ for SF12) and during the payload time (30 and $55 \mathrm{~ms}$ for SF7 and 1 and $1.5 \mathrm{~s}$ for SF12). The measurement period is shown for each technology in Figure 2.

In case of SigFox interference testing, due to long transmission time, the measurement period was longer implying the need for longer measurements for each case. The measurement time in SigFox interference case was 16 minutes while for the other two technologies it was 4 minutes per measurement. This allowed to collect around 100 packets per measurement that were enough to draw statistics in terms of percentage of packets lost and received with wrong payload CRC. For each interfering technology there were in total 16 different measurements, for each combination of 4 time shifts, 2 different power levels and 2 different spreading factors.

\section{B. Technology emulation}

As we did not have the Z-Wave and IO Home Control end nodes we emulated the packet transmission using Zolertia Remote [18]. The Zolertia Remotes use the CC1200 chip from Texas Instruments for sub-GHz communication and can support modulations that are used for Z-Wave and IO Home Control, GSFK and 2-FSK, respectively. It has a programmable bandwidth from $12.5 \mathrm{kHz}$ to $1600 \mathrm{kHz}$ offering data rates in the range $1.2 \mathrm{kbps}$ to $1250 \mathrm{kbps}$. They are flashed with the Time Annotated Instruction Set Computer (TAISC) framework [19] based on the Contiki operating system. This setup runs a custom program to transmit emulated Z-Wave/IO Home Control packets as soon as one of the input pins of the Zolertia is toggled. In case of Z-Wave, the RF profile R3 was emulated as only that profile interferes with the LoRa channel at $868.3 \mathrm{MHz}$ center frequency.

Differently from Z-Wave and IO Home Control case, in SigFox case we used an TDnext RF module to transmit uplink SigFox packets [2]. TD1207R devices offers a receiver sensitivity of $-126 \mathrm{dBm}$ and an output power of $+16 \mathrm{dBm}$, which makes these devices compatible with the Sigfox ${ }^{T M}$ network. The TD1207R radios are not possible to be triggered via microcontroller (as it is required in our setup) but they can be operated via AT commands (triggered by a computer). As precise timing of transmission is required, the TD1207R is triggered by AT commands that are generated by a Zolertia Remote [18], while the Zolertia itself is triggered by the microcontroler. The SigFox module was configured to operate on randomly allocated SigFox channels.

The time and frequency illustration of the technologies is shown in Figure 3. It is seen from the spectrograms that Sigfox (Figure 3a) has the longest time-on-air compared to the shortest just some ms for Z-Wave (Figure 3c).

\section{Results}

This section will detail the achieved results for the interference impact of other sub-GHz technologies in LoRa.

Results when LoRa uses SF7 are given in Table III. The most severe interference is when it happens during the preamble time (time shift of $8 \mathrm{~ms}$ ). This is from the fact that due to interference the receiver can not get synchronized with the transmitter by losing the preamble start, making thus impossible for the LoRa receiver to start receiving the packet. For the time shift of $17 \mathrm{~ms}$ (when interference starts during the header time) losses are less compared to the preamble time shift but higher than in payload. As during the measurements explicit physical headers for LoRa were being used, if the header CRC is wrong the receiver will stop receiving the packet. This will cause the packet to be lost even if the interference is short and happens only during the header time. This is the case with Z-Wave interference when the interfering 


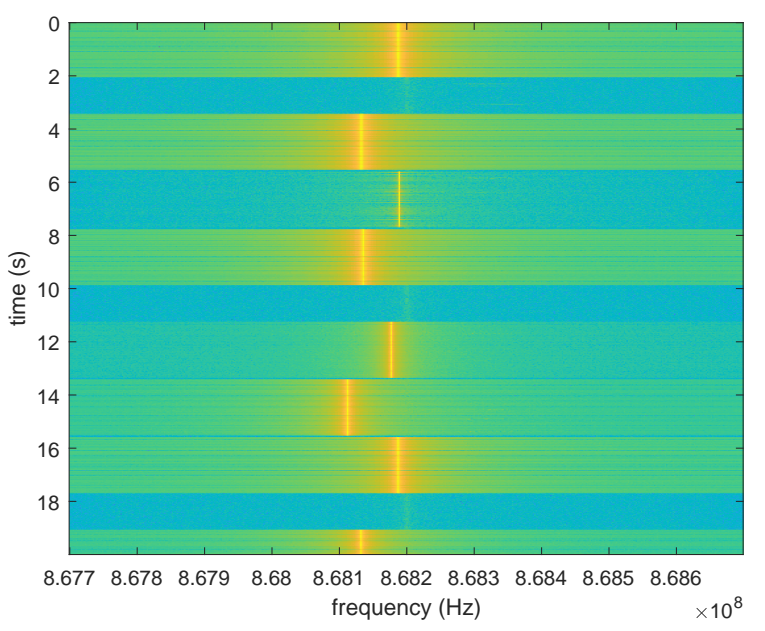

(a) Sigfox

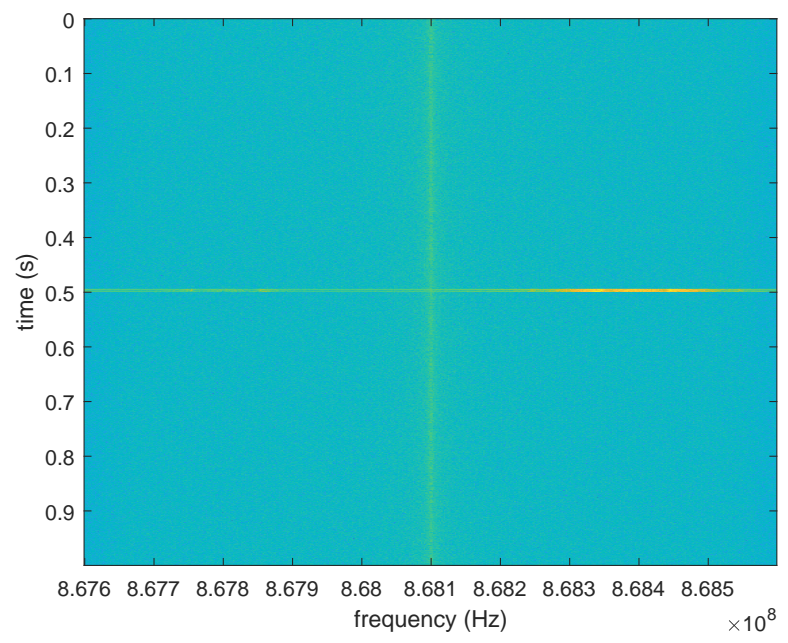

(c) Z-Wave

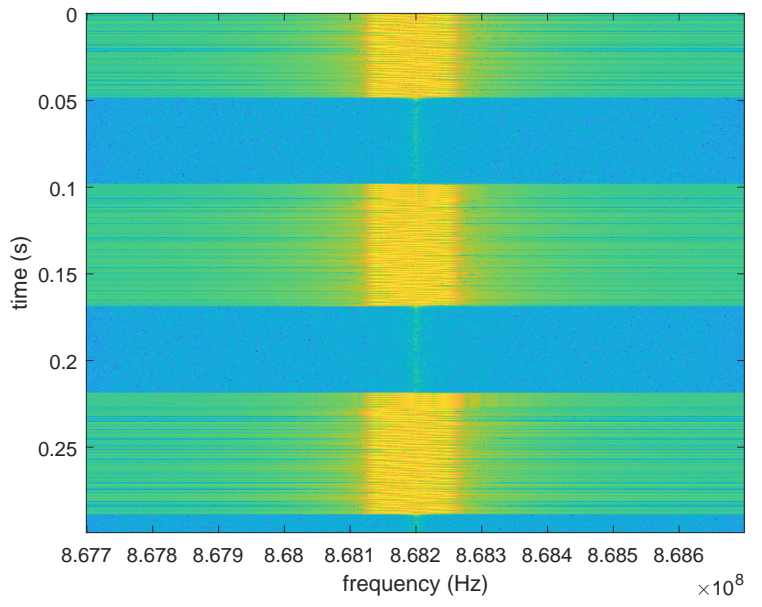

(b) LoRA - SF7

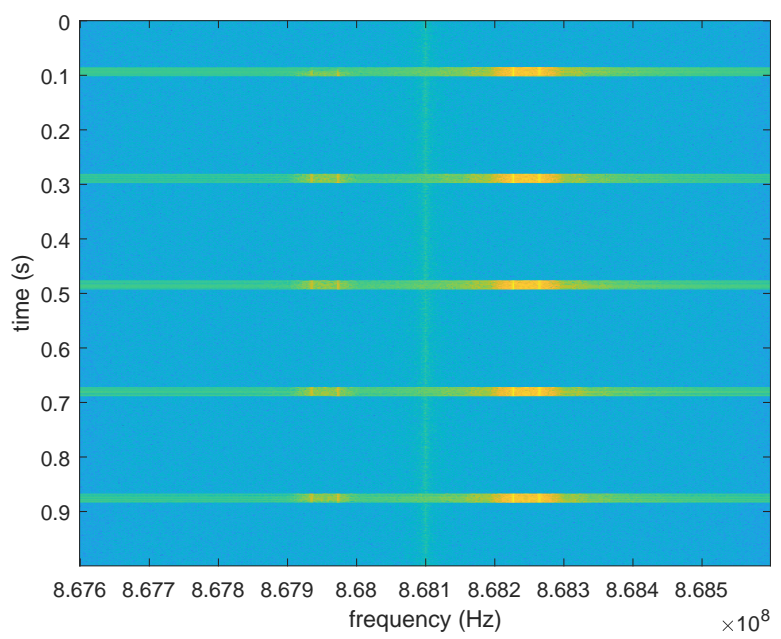

(d) IO Home Control

Fig. 3. Non-overlap transmission of sigfox, LoRA - SF7, zwave, and iohome.

time is not longer than $5 \mathrm{~ms}$. Comparing between different technologies, SigFox causes the highest packet losses for LoRa due to its long burst transmission even though it uses narrow bands. This will make impossible for LoRa to recover as the interference is longer than the LoRa packet itself. In case of Z-Wave and IO Home Control interference time is shorter (5 $\mathrm{ms}$ and $15 \mathrm{~ms}$, respectively). That has practically no impact if it starts during the payload time as it can be recovered by using FEC codes in LoRa.

Considering the usage of SF12 the results are shown in Table IV. In general, for each measurement case losses are lower than when SF7 was used. This is due to lower receiver sensitivity for SF12 as well as the ability to receive packets in lower SNR threshold, $-22 \mathrm{~dB}$, compared to $-7.5 \mathrm{~dB}$ for SF7 [9]. Even for SF12 the losses based on different technologies and different time shifts does not make any exception compared to SF7 case. The most problematic technology is SigFox as
TABLE III

PERCENTAGES OF PACKET LOSSES FOR DIFFERENT INTERFERING TECHNOLOGIES, DIFFERENT POWER LEVELS AND DIFFERENT TIME SHIFTS. LORA IS CONFIGURED TO USE SF7

\begin{tabular}{|c||c|c|c|c|}
\hline Interfering tech - Time shifts & $\mathbf{8} \mathbf{~ m s}$ & $\mathbf{1 7} \mathbf{~ m s}$ & $\mathbf{3 0} \mathbf{~ m s}$ & $\mathbf{5 5} \mathbf{~ m s}$ \\
\hline \hline SigFox $-10 \mathrm{~dB}$ & $15 \%$ & $16 \%$ & $14 \%$ & $9 \%$ \\
\hline SigFox $+10 \mathrm{~dB}$ & $28 \%$ & $18.80 \%$ & $14 \%$ & $12 \%$ \\
\hline Z-Wave $-10 \mathrm{~dB}$ & $16 \%$ & $3 \%$ & $0 \%$ & $0 \%$ \\
\hline Z-Wave $+10 \mathrm{~dB}$ & $17 \%$ & $1.80 \%$ & $2 \%$ & $0 \%$ \\
\hline IOHomeControl -10 dB & $12.3 \%$ & $1.50 \%$ & $0 \%$ & $0 \%$ \\
\hline IOHomeControl $+10 \mathrm{~dB}$ & $12.8 \%$ & $2.6 \%$ & $0.5 \%$ & $0 \%$ \\
\hline
\end{tabular}

it has the highest impact on LoRa packet losses compared to other technologies. Regarding different time shifts, even in this case, the most severe case is when interference starts during the preamble time. 
TABLE IV

PERCENTAGES OF PACKET LOSSES FOR DIFFERENT INTERFERING TECHNOLOGIES, DIFFERENT POWER LEVELS AND DIFFERENT TIME SHIFTS. LORA IS CONFIGURED TO USE SF12

\begin{tabular}{|c||c|c|c|c|}
\hline Interfering tech - Time shifts & $\mathbf{1 5 0} \mathbf{~ m s}$ & $\mathbf{4 0 0} \mathbf{~ m s}$ & $\mathbf{1 ~ s}$ & $\mathbf{1 . 5} \mathbf{~ s}$ \\
\hline \hline SigFox $-10 \mathrm{~dB}$ & $14 \%$ & $7.35 \%$ & $12 \%$ & $6 \%$ \\
\hline SigFox $+10 \mathrm{~dB}$ & $20 \%$ & $20 \%$ & $14 \%$ & $8 \%$ \\
\hline Z-Wave $-10 \mathrm{~dB}$ & $5 \%$ & $2 \%$ & $0 \%$ & $0 \%$ \\
\hline Z-Wave $+10 \mathrm{~dB}$ & $7 \%$ & $2.00 \%$ & $0 \%$ & $0 \%$ \\
\hline IOHomeControl -10 dB & $7.2 \%$ & $1.50 \%$ & $0 \%$ & $0 \%$ \\
\hline IOHomeControl $+10 \mathrm{~dB}$ & $12.4 \%$ & $1 \%$ & $0 \%$ & $0 \%$ \\
\hline
\end{tabular}

As it is shown in Tables III and IV the worst case scenario in terms of time shifts is when the interferer starts during the preamble time. However, the impact of power level is not straight forward noticeable. For this we do a set of measurements only for time shifts when the interferer starts during preamble time, but adjusting the received signal level difference between LoRa transmission and interferer.

The packet loss ratio for Z-Wave interferer case is shown in Figure 4a. It is seen that if LoRa signal will be lower than 20 $\mathrm{dB}$ compared to Z-Wave signal, the packet loss will be higher than $50 \%$, reaching $96 \%$ for $-25 \mathrm{~dB}$ difference in case of SF7. On the other hand, for SF12 even for difference of $-25 \mathrm{~dB}$ in signal level packet losses are still only $15 \%$. This comes due to the higher sensitivity of SF12 that is able to receive signals even at SNR level of $-22 \mathrm{~dB}$. In case when LoRa signal is much stronger than Z-Wave, the losses are around $6 \%$ for SF12 and higher for SF7 (up to 16\%). These residual losses are due to the fact that the power level of the Z-Wave is fixed at $-65 \mathrm{dBm}$ that impacts LoRa receiver. We would expect lower impact if the Z-Wave power level is lower, e.g. at -80 to $-100 \mathrm{dBm}$.

Sigfox interferer case is shown in Figure $4 \mathrm{~b}$. When LoRa signal is lower than $20 \mathrm{~dB}$ compared to SigFox signal, the packet losses increase fast and can be as high as 67\% for SF7 at $-25 \mathrm{~dB}$ signal difference. By increasing the Sigfox signal level at the receiver site, the packet loss ratio is increased faster than when the interferer was Z-Wave emulated transmitter. However, in this case the differences between using SF7 and SF12 are not that big as in case of Z-Wave. This comes as result of long Sigfox transmission that will impact even SF12 long packet transmission during the whole packet time as in case of SF7.

IO Home Control interferer case is shown in Figure 4c. For LoRa signal $20 \mathrm{~dB}$ lower than IO Home Control signal at the receiver side the packet loss will be around $22 \%$ for SF7 and $14 \%$ for SF12. The differences between SFs come as result of the different receiver sensitivities and ability to receive packets even under noise level. On the other hand, when LoRa is more than $20 \mathrm{~dB}$ stronger than IO Home Control interferer the losses are less than $10 \%$ for both SFs.

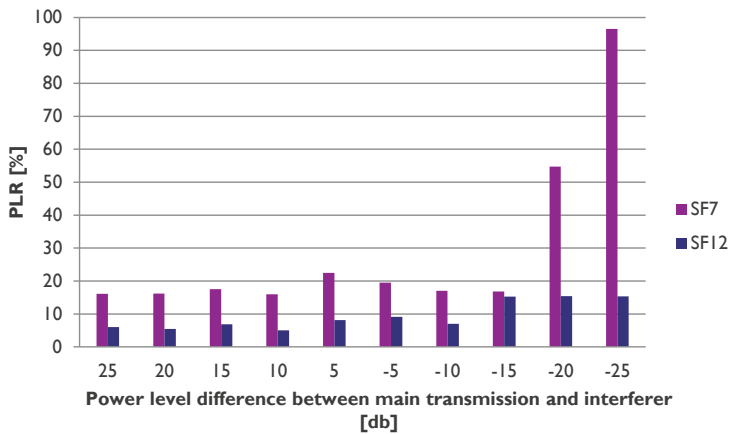

(a) Z-Wave interferer

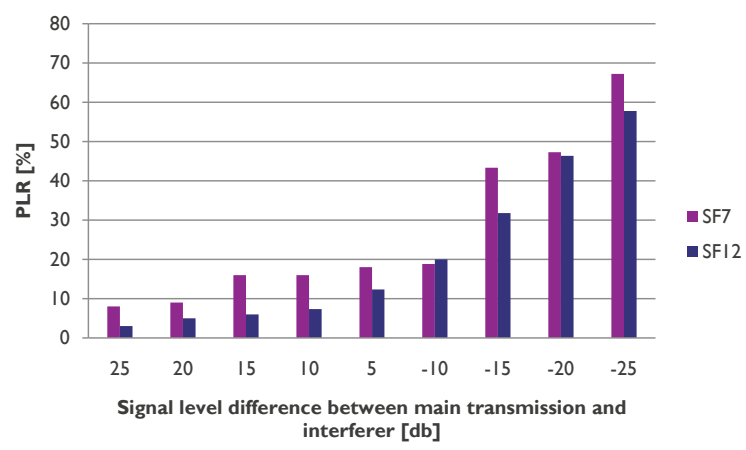

(b) Sigfox interferer

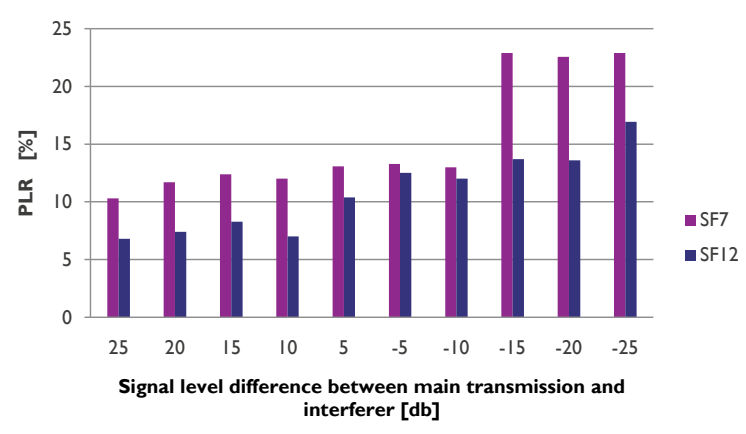

(c) IO Home Control interferer

Fig. 4. Packet loss ratio for different received signal levels between LoRa and interferer signal when the interferer signal starts during the preamble time.

\section{CONCLUSIONS AND FUTURE WORK}

In this paper we describe a controllable measurement setup for interference testing between different technologies. Further, we perform interference measurements to evaluate the impact of other sub-one $\mathrm{GHz}$ technologies (SigFox, Z-Wave, IO Home Control) in LoRa. Based on the results it is shown that if the interference starts during the preamble time, losses can be as high as $28 \%$ in case of SigFox interference. Losses are reduced, becoming zero, if the interference starts by the end of the payload, no matter the interfering technology. A slightly higher losses $(+5$ to $+10 \%)$ are recorded when SF7 is used instead of SF12 for the same cases.

Using the same setup future measurement can be done for different power levels as well encountering more transmitters at the same time. 


\section{ACKNOWLEDGMENT}

This research was funded by the Flemish FWO SBO S004017N IDEAL-IoT (Intelligent DEnse And Long range IoT networks) project.

\section{REFERENCES}

[1] D. Micheli, A. Delfini, F. Santoni, F. Volpini, and M. Marchetti, "Measurement of electromagnetic field attenuation by building walls in the mobile phone and satellite navigation frequency bands," IEEE Antennas and Wireless Propagation Letters, vol. 14, pp. 698-702, 2015.

[2] https://s3-eu-west 1.amazonaws.com/assetstdnext/download/TDnext+RF, Last visited January 2018.

[3] B. Reynders, W. Meert, and S. Pollin, "Range and coexistence analysis of long range unlicensed communication," in Telecommunications (ICT), 2016 23rd International Conference on. IEEE, 2016, pp. 1-6.

[4] C. Goursaud and J.-M. Gorce, "Dedicated networks for iot: Phy/mac state of the art and challenges," EAI endorsed transactions on Internet of Things, 2015.

[5] D. Croce, M. Gucciardo, I. Tinnirello, D. Garlisi, and S. Mangione, "Impact of spreading factor imperfect orthogonality in lora communications," in International Tyrrhenian Workshop on Digital Communication. Springer, 2017, pp. 165-179.

[6] C. Orfanidis, L. M. Feeney, M. Jacobsson, and P. Gunningberg, "Investigating interference between lora and ieee 802.15. 4g networks," in 2017 IEEE 13th International Conference on Wireless and Mobile Computing, Networking and Communications (WiMob). IEEE, 2017, pp. 1-8.

[7] K. Mikhaylov, J. Petäjäjärvi, and J. Janhunen, "On lorawan scalability: Empirical evaluation of susceptibility to inter-network interference," in Networks and Communications (EuCNC), 2017 European Conference on. IEEE, 2017, pp. 1-6.

[8] J. Haxhibeqiri, A. Karaagac, F. Van den Abeele, W. Joseph, I. Moerman, and J. Hoebeke, "Lora indoor coverage and performance in an industrial environment: Case study," in Emerging Technologies and Factory Automation (ETFA), 2017 22nd IEEE International Conference on. IEEE, 2017, pp. $1-8$

[9] "Sx1272/73860 mhz to $1020 \mathrm{mhz}$ low power long range transceiver," Available online: http://www.semtech.com/images/datasheet/sx1272.pdf, Last visited January 2018.

[10] O. B. SELLER and N. Sornin, "Low power long range transmitter," Feb. 2 2016, uS Patent 9,252,834.

[11] "Sigfox: The global communications service provider for the internet of things (iot)," https://www.sigfox.com/en.

[12] B. Vejlgaard, M. Lauridsen, H. Nguyen, I. Kovács, P. Mogensen, and M. Sørensen, "Coverage and capacity analysis of sigfox, lora, gprs, and nb-iot," in Proceedings of the IEEE 85th Vehicular Technology Conference, Sydney, Australia, 2017, pp. 4-7.

[13] "Series g: Transmission systems and media, digital systems and networks, access networks in premises networks, short range narrow-band digital radiocommunication transceivers phy and mac layer specifications," G.9956, TELECOMMUNICATION STANDARDIZATION SECTOR OF ITU, 2012.

[14] http://www.analog.com/media/en/technical-documentation/data sheets/ADF7022_2page.pdf, Last visited February 2018.

[15] "Attenuator unit 0,5-6,0 ghz pah-6000/80-8." Available online: http://doc.ilabt.iminds.be/ilabtdocumentation/_static/qosmotec/Attenuator_meetresultaten.PDF, Last visited January 2018.

[16] "Splitter unit." Available online: http://doc.ilabt.iminds.be/ilabtdocumentation/_static/qosmotec/Splitter_Unit.PDF, Last visited January 2018.

[17] J. Haxhibeqiri, F. Van den Abeele, I. Moerman, and J. Hoebeke, "Lora scalability: A simulation model based on interference measurements," Sensors, vol. 17, no. 6, p. 1193, 2017.

[18] https://github.com/Zolertia/Resources/wiki/RE Mote, Last visited February 2018.

[19] B. Jooris, J. Bauwens, P. Ruckebusch, P. De Valck, C. Van Praet, I. Moerman, and E. De Poorter, "Taisc: a cross-platform mac protocol compiler and execution engine," Computer Networks, vol. 107, pp. 315326, 2016. 